\title{
Optimized duration of antituberculosis treatment for managing female genital tuberculosis: real-world experience from a high prevalence region in Eastern China
}

Jianghua Yang ( $\nabla$ yjhpathway@163.com )

First affiliated Hospital of Wannan Medical College

Jian Sun

First affiliated Hospital of Wanan Medical College

Wenjie Wang

First affiliated Hospital of Wannan Medical College

Jing Ding

First Hospital of Wannan Medical College

\section{Yusheng Cheng}

First Affiliated Hospital of Wannan Medical College

\section{Yunfeng Zhou}

First Affiliated Hospital of Wannan Medical College

\section{Research article}

Keywords: female genital tuberculosis, tuberculous peritonitis, antituberculosis therapy, directly observed therapy, duration of treatment

Posted Date: August 13th, 2020

DOI: https://doi.org/10.21203/rs.3.rs-55589/v1

License: (c) (i) This work is licensed under a Creative Commons Attribution 4.0 International License. Read Full License 


\section{Abstract \\ Background}

Duration of antituberculosis therapy (ATT) for managing female genital tuberculosis (FGTB) is controversial with the intermittent regimen no more advocated. We therefore conducted a prospective, real-world research to compare 6 months and 9 months of ATT.

\section{Methods}

Between 2012 and 2018, 109 drug-susceptible patients newly diagnosed with FGTB and/or tuberculous peritonitis (genital, 13; peritoneal, 34; mixed, 62) received naïve treatment for 9-12 months and further 18-month follow-up. Data on disease features at baseline and long-term outcome (intent-to-treat) were compared between group A (aged 15-35 years) and group B (aged $\geq 36$ years). Efficacy and side effects of treatment were compared within each group 6 months and 9 months from ATT initiation (per-protocol), respectively.

\section{Results}

In contrast to group B at baseline, group A had more clinical evidence predicting active tuberculosis ( $P<$ 0.05), severer performance of genital lesions and pelvic adhensions $(P<0.05)$, more signs of active pulmonary tuberculosis $(P<0.01)$, and less performance of only TBP $(P<0.01)$. Intent-to-treat analysis showed higher incidence of overall single side effects and poor compliance in group $B(P<0.05)$, and similar recurrence rate between 2 groups. Per-protocol analysis showed increased complete response rate $(P<0.01)$ and similar incidence of side effects $(P>0.05)$ in group $A$, similar complete response rate $(P>$ $0.05)$ and increased incidence of overall single side effects $(P<0.05)$ in group $B$ at 9-mo duration.

\section{Conclusions}

Younger females with FGTB had a greater risk of systemic infection of TB compared to older ones. Ninemonth ATT using daily therapy proved to be beneficial for younger patients at reproductive age. Sixmonth option was suitable for older patients for improving the side effects and poor compliance in the duration of treatment.

\section{Introduction}

Female genital tuberculosis (FGTB), an important type of extra-pulmonary tuberculosis (EPTB), is caused by the infection of mycobacterium tuberculosis (MTB) mainly disseminated from pulmonary tuberculosis (PTB) [1]. With continuous prevalence of TB and increased immigrants [2], FGTB causes significant morbidity, atypical clinical symptoms and multiple sequelae in infected females [1, 3-5]. 
Six-month antituberculosis therapy (ATT) using directly observed therapy (DOT) is the globally accepted treatment for drug-susceptible tuberculosis (DS-TB) $[2,6]$. However, the duration of managing FGTB or abdominal TB continues to be controversial especially when the intermittent or shorter-course regimen, showing a high risk of treatment failure and disease relapse [7-10], is no more advocated by World Health Organization (2017 update guidelines for treatment of DS-TB) [6]. Despite 6-month ATT showing objective efficacy for managing FGTB or abdominal TB in some randomized controlled trials excluding older patients or patients with comorbidity of other types of TB $[11,12]$, there is lack of data especially real-world data supporting its efficacy. It brings a new debate, how to make a simplified, optimized strategy of ATT, for managing FGTB in patients at different age categories especially those with comorbidity of other types of TB and/or immune-deficient diseases.

We therefore conducted an open, real-world study to evaluate its efficacy using daily therapy in a large group of consecutive treatment-naive patients with FGTB. A secondary objective was to compare the disease expression, side effects and patients' compliance among patients at different age categories.

\section{Patients And Methods}

\section{Patients}

All eligible females (aged $\geq 15$ years) between 2012 and 2018, who were newly diagnosed as either FGTB or tuberculous peritonitis (TBP) in the region, were included in the study. In the specific period, 127 consecutive females were diagnosed in our center with the disease. Among them, 7 patients unclearly diagnosed after re-evaluation, 9 patients suspected with DR-TB and additional 2 elderly patients with comorbidity of malignancy, were ineligible for receiving standard treatment and excluded. The remaining 109 patients were divided into group A (younger females at reproductive age, aged 15-35 years) and group $B$ (older females at reproductive age and postmenopausal period, aged $\geq 36$ years) (Figure 1).

\section{Clinical data collection}

Collected clinical data included epidemiological and clinical characteristics, hematological and biochemical tests, and Tuberculin Skin Test (TST) or T-SPOT.TB (T-SPOT.TB replaced TST from 2015). All patients took chest x-ray and 95 underwent chest computerized tomography (CT) scan during hospitalization. Patients with comorbidity of PTB, either active or non-active, were carefully evaluated with imaging data stored in our electronic image system whenever needed. Fourty-two patients underwent laparotomy, laparoscopy or hysteroscopy. Surgical and histological findings were carefully recorded. Ultrasound-guided peritoneocentesis was performed in 59 of 86 patients with performance of ascites. Data of ascitic tests, including routine and biochemical tests, adenosine deaminase (ADA), acidfast bacilli (AFB) on smear or culture, were also recorded. Positive definition of TST followed National Guidelines of Tuberculosis Diagnosis (WS 288-2017, China). Operative procedures and positive definition of T-SPOT.TB followed instructions of T-SPOT.TB assay kit (Oxford Immunotec, Abingdon, UK). According to relevant reports to T-SPOT.TB $[13,14]$ and its efficacy for interpreting active TB in the region, a response was considered stronger (moderate-to-strong) positive in this study if the number of spots per 
test well was $\geq 16$ (when the background control count was $<5$ ), or at least triple the value found in the background control wells (when the background control count was $\geq 5$ ).

\section{Diagnostic criteria for FGTB and TBP}

A "definite" diagnosis of FGTB was considered in the presence of $\geq 2$ of the following: ( $\nabla$ ) epidemiological, clinical, and imaging or surgical evidence of FGTB involvement; ( () positive result of AFB on smear/culture; ( $(\mathbb{Z})$ stronger positive TST/T-SPOT.TB, ( $(\mathbb{)})$ histopathological presence of TB granulomas. Presumptive diagnosis of FGTB was made if there was strong clinical suspicion, such as clinical or explorative features, genital lesions (tubo-ovarain mass) and/or PTB on imaging, and clinically confirmed when the diagnostic ATT proved to be effective.

A "definite" diagnosis of TBP was based on the presence of high-protein (>2.5 g/dL) ascites containing $>250$ white blood cells $/ \mathrm{mm}^{3}$ along with $\geq 2$ of the following: $(\mathbb{\nabla})$ imaging evidence of peritoneal inflammation; ( $(\mathbb{)})$ ascetic ADA levels $>35 \mathrm{u} / \mathrm{L}(\mathbb{\nabla})$ stronger positive TST/T-SPOTTB; ( () positive AFB on smear/culture or presence of TB granulomas in the peritoneal biopsies. Presumptive diagnosis of TBP was smilar to FGTB (except genital lesions).

\section{ATT protocol and management of side effects}

Intensive phase included 4 drugs (isoniazid $300 \mathrm{mg}$, rifampicin 450mg, pyrazinamide $1250 \mathrm{mg}$, and ethambutol $750 \mathrm{mg}$ ) daily for 2 months. Patients weighing $>50 \mathrm{~kg}$ received additional doses of rifampicin, pyrazinamide and ethambutol (150 mg, $250 \mathrm{mg}$ and $250 \mathrm{mg}$, respectively). The continuation phase included isoniazid and rifampicin for 7-10 months according to individual response (9-12 months' duration widely taken in EPTB management in the specific period). Drug-induced side effects were all recorded and managed in comprehensive measures relevant to individual performance.

\section{Follow-up and compliance to Treatment}

Patients were registered and followed up at regular intervals at TB clinics of our center. DOT was administered by patients' family members (trained by professional physicians) in the region. Whole blood tests, liver function tests, chest x-ray or chest CT, and abdominal ultrasonic scan/ CT were done per month in the intensive phase and every 2-3 months in the duration phase. Patients who reduced doses of drug-intake for side effects received symptomatic treatment after careful counseling. Patients who took irregular protocol (taking drugs $<80 \%$ of intended days, or stopped drug-intake under no guidance) or lost to follow-up were defined as poorly compliant patients and excluded on intent-to-treat analysis.

\section{Assessment at two observation time-points}

Based on the aim of the study, the efficacy of treatment was assessed in 109 patients at 6 months and 9 months from the initiation of ATT respectively (per-protocol analysis). All patients underwent clinical evaluation and abdominal-pelvic imaging to demonstrate healing of lesions. Chest CT scan and AFB smear/culture of sputum were repeated in those who presented with PTB and regarded as an important 
measure to demonstrate the response to treatment. Twenty-three patients were persuaded to repeat hysteroscopy and/or endometrial biopsy. Surgical and histopathological findings were recorded for assessment.

\section{Outcome measures}

"Complete clinical response" was defined as complete resolution of symptoms and signs, normalization of biochemical and hematological tests, and healing of TB lesions on imaging or repeat surgical procedures in this study. A partial response was defined as resolution of clinical manifestations and partial healing of TB lesions. Non-response was defined persistent clinical symptoms, persistence of TB granuloma on histopathology or AFB on microscopy/culture, persistence of TB lesions on imaging or repeat surgical procedures. Healing of PTB referred to healing of active PTB lesions (miliary tubercles, cavity, infiltrated lesions and tuberculous pleural effusion) along with no progression of non-active PTB.

\section{Recurrence during 18-month follow-up}

Eighty-eight patients persisted for 9-month treatment. Patients with clinical response were followed up 6monthly for 18 months at TB clinics of our center, and those with no response every 1-3 months. Those who failed to visit the clinics were contacted telephonically and interviewed for recurrence. To compare the disparity of recurrence rate between 2 groups on intent-to-treat analysis, patients who lost to follow-up were defined as individuals with no recurrence.

\section{Statistical Analysis}

Statistic analysis was conducted using SPSS version 22.0 (IBM, Armonk, New York, USA). Parametric results are expressed as mean (range) where appropriate. Non parametric category of variables was analyzed by $\chi^{2}$ test, $\chi^{2}$ with Yate's correction or Fisher's Exact test, where applicable. Two-sided $P<0.05$ was considered as statistically significant.

\section{Results}

\section{Characteristics of the patients at baseline}

The main epidemiological, clinical and laboratory characteristics of 109 patients at baseline are shown in Table 1. Recent pregnancy or reproduction (31.7\%:2.9\%, $P<0.001)$, infertility (36.6\%:11.8\%, $P=0.002)$, ascitic ADA>35 u/L (88.2\%:47.6\%, $P=0.010)$, and stronger positive TST (66.7\%:23.3\%, $P=0.022)$ or T.SPOTTB (96.4\%:64.9\%, $P=0.006)$ were all at higher incidence in Group A, whereas Group B had more presentation with immune-deficient diseases (16.2\%:2.4\%, $P=0.057)$ and obvious ascites (60.3\%:22.0\%, $P<0.001)$. There was no difference in others of the above parameters between 2 groups at baseline. 17 $(41.5 \%)$ patients of group A and 27 (39.7\%) of group B underwent surgical exploration respectively. Typical performance of FGTB, including thickening-stiff or tortuous tubes (76.5\%:33.3\%, $P=0.013)$, 
hydrosalpinx or pyosalpinx (29.4\%:3.7\%, $P=0.049)$, and pelvic adhesions $(82.4 \%: 44.4 \%, P=0.030)$ were more seen in Group A, but no difference seen in other surgical or histopathological parameters (Table 2).

\section{Predominant TB sites of disease and comorbidity of other TB sites}

FGTB or TBP was definitively diagnosed in $38(92.7 \%)$ patients of group $A$ and 60 (88.2\%) of group B, respectively. The remaining 11 patients were presumptively diagnosed and clinically confirmed. Of 41 patients in group A, $6(14.6 \%)$ had FGTB, 5 (12.2\%) had TBP, and $30(73.2 \%)$ had features of both. Of 68 patients in group B, $7(10.3 \%)$ had FGTB, $29(42.6 \%)$ had TBP, and $32(47.1 \%)$ had both. Simple performance of TBP was at higher incidence in Group B in contrast to group A (42.6\%:12.2\%, $P=0.004)$. Both groups showed similar incidence of non-active PTB (26.8\%:42.6\%, $P>0.05)$ and other EPTB (19.5\%:25.0\%, $P>0.05$ ), but active PTB was more seen in group A (43.9\%:14.7\%, $P=0.009$ ) (Table 3 ).

\section{Intent-to-treat anlaysis}

Twenty-one patients were lost to follow-up or took irregular protocol in 9-month duration after initiation of treatment. Of 12 patients (11 in group B) who took irregular protocol (almost all declined further consultation at TB clinics), 9 stopped drug-intake under no guidance and 3 took drugs $<80 \%$ of intended days. Of 9 patients lost to follow-up, 7 were from group B. Intent-to-treat analysis showed that Group B had higher incidence of poor compliance (26.5\%:7.3\%, $P=0.027)$, and higher incidence of DILI (20.6\%:4.9\%, $P=0.049)$ and overall single side effects (42.6\%:17.1\%, $P=0.006)$ than group A. In addition, group B also showed higher incidence of anorexia (26.5\%:12.2\%), vomiting (14.7\%:7.3\%) and leucopenia/ thrombocytopenia $(7.4 \%: 2.4 \%)$ than group $A$, but there was no significant difference in statistic analysis $(P>0.05)$.

\section{Per-protocol analysis}

Excluding 21 poorly compliant patients, the remaining 88 patients who persisted for $\geq 9$ months' ATT were taken on per-protocol analysis (Table 5). Increased complete response was achieved in group A at 9month time-point compared to their performance at 6-month one $(92.1 \%: 63.2 \%, P=0.006)$, whereas no disparity was shown in group $\mathrm{B}(88.0 \%: 87.7 \%, P>0.05)$.

Equal side effects, either single or overall, were shown in Group A between two time-points (10.5\%:15.8\%, $P>0.05)$, whereas incidence of anorexia $(26.0 \%: 8.8 \%, P=0.016)$, DILI $(22.0 \%: 7.0 \%, P=0.033)$ and overall single side effects (42.0\%:22.8\%, $P=0.033$ ), significantly elevated in group $B$ at 9-month time-point compared to their performance at 6-month one. Despite no disparity, either single or overall side effects, was shown between 2 groups at the 6-mo time-point $(P>0.05)$, group $\mathrm{B}$ had higher incidence of overall single side effects than group $A$ at 9 -mo time-point $(42.0 \%: 15.8 \%, P=0.008)$. None died during the period of treatment.

\section{8-month follow-up for recurrence}


During 18-month follow-up after 9-month duration, 7 patients (2 from group A and 5 from group B) were lost to follow up; 4 patients ( 1 from group $A$ and 3 from group $B$ ) had recurrence of disease after standard treatment. Recurrence also occurred in additional 3 patients from group $B$ who took irregular protocol within 6-mo duration. Of 6 patients with TB recurrence from group B, 3 had comorbidity of diabetes and lost control of blood glucose around the recurrence period; 2 had decompensated liver cirrhosis; 1 had sicca syndrome and long-term use of glucocorticoids. They were all considered as DS-TB, retreated with DOT category $\otimes$ protocol for 6-9 months along with moderate doses of drugs and symptomatic treatment, and all responded.

The patient (aged 32 years at enrollment) with recurrence was diagnosed with DR-TB after careful reevaluation, retreated with DOT category $\otimes$ protocol for $>18$ months, received bilateral salpingectomy (denied by her family members last time) after completing 6-month intensive phase, and responded (in the continuation phase before the study completed). No recurrence was observed in the rest of the patients (except those lost to follow-up) at the end of 18-month follow-up.

\section{Discussion}

The present open real-world observational study assessed the natural history of FGTB in a cohort of consecutive drug-susceptible patients followed in a region of Eastern China for a long period. As FGTB usually co-occurs with TBP, eligible females newly diagnosed with FGTB or TBP in the region were all recruited for better analyzing the composition of disease. 41 (37.6\%) of 109 patients were younger females at reproductive age. Of 68 older patients, 25 (36.8\%) were older females at reproductive age (aged 36-49 years) and 43 (63.2\%) were postmenopausal females (aged $\geq 50$ years). Both of them were included in group B for no obvious discrepancy (disease expression or efficacy of treatment or side effects) found. Special emphasis was given in the association between the severity of disease and age composition of patients, and more specifically on the link between duration of daily ATT and outcome of patients.

Three major points are raised in this study: $(\mathbb{\nabla})$ disease expression and compliance to treatment are mainly dependent of age; ( $($ ) 6-month ATT brings more benefits for older patients (similar response rate and fewer side effects); ( $($ ) 9-month ATT using daily therapy proves to be a better option for younger patients at reproductive age (similar side effects but increased complete response rate).

The mean age of developing FGTB is 40 year in developed nations [3, 5], whereas the disease presents in younger age group (20-30 year) in Asia due to early marriage and child-bearing in them $[1,4,15,16]$. This study confirms that FGTB can affect patients at any age with a peak incidence and severe disease presentation in younger females at reproductive age (15-35 year). Of 75 females diagnosed with FGTB (or mixed with TBP) in the study, 36 (48.0\%) were younger females (aged 18-34 year) and $13(36.1 \%)$ of them had pregnancy or reproduction within 1 year before onset of disease, confirming that FGTB more likely occurs in younger females who had increased blood supply in genital tract and/or damaged genital tract barrier due to invasive procedures $[1,16]$. Interestingly, despite FGTB possibly omitted in some 
patients especially older ones ( 29 of 68 presented with only TBP) who declined surgical exploration, both composition and expression of disease seemingly turned milder with ascending age, indicating a closed link between haematogenous transmission of MTB and age of females. It was also verified in the

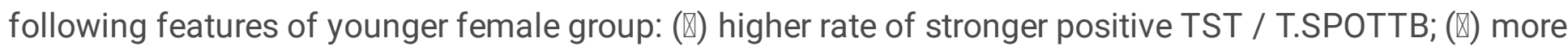
performance of typical FGTB; $(\mathbb{\nabla})$ higher incidence of comorbidity of active PTB.

Poor compliance, a major problem in managing TB and closely associated with side effects during treatment, may cause a series of adverse consequences including prolonged course of disease, drug resistance and recurrence $[2,6,17-19]$. Six-month ATT, especially intermittent 6-month therapy or even shorter one, therefore, mainly aims to manage the problem $[7,8,10,11,17]$. This study indicates that older patients likely had higher incidence of poor compliance and side effects especially digestive symptoms and DILI (commonly seen in the region with high-burden of hepatitis B) in the longer duration compared to younger patients, whereas the efficacy of 6-month and 9-month ATT were equal. Although recurrence of disease occurred in 6 patients ( 3 obeyed to protocol and 3 not) with uncontrolled immunodeficient diseases during 18-month follow-up, they were all diagnosed with DS-TB and responded to retreatment after controlling respective comorbidity. According to 2017 update guidelines of WHO [6] and the results of this study, six-month ATT using daily therapy proved to be an appropriate option for managing FGTB in older females especially those with comorbidity of immuno-deficient diseases.

In contrast to older patients, 38 (92.7\%) younger patients persisted for 9-month duration along with similar incidence of side effects and increased complete response rate. Of 29 (70.7\%) younger patients with comorbidity of PTB, 8 (miliary PTB and infiltrating PTB mixed with tuberculous pleuritis presented in 6 and 2 patients, respectively) only achieved a partial healing of PTB lesions after 6 -month treatment, but all achieved obvious healing after 9-month treatment, indicating that longer duration of ATT is helpful for managing systemic infection of TB. Nine-month ATT using daily therapy, therefore, may be a better option for managing FGTB in younger females at reproductive age.

There are a few weaknesses needed to mention in the study. Firstly, most of the older patients declined to receive or repeat hysteroscopy or endometrial biopsy for economic or personal reasons. It possibly reduced the diagnostic rate of FGTB in those presenting with simple performance of TBP, and not beneficial for accurate assessment of healing of FGTB lesions. Furthermore, AFB on polymerase chain reaction, beneficial for early diagnosis and efficacy assessment of FGTB treatment especially when typical TB granulomas was found in endometrium $[12,16,20]$, was not taken in the study for our lacking of equipments in the specific period.

\section{Conclusions}

The results of this study demonstrate that younger females with FGTB, in contrast to older ones, had a higher risk of systemic infection of TB and better compliance to ATT using daily therapy. Duration of ATT for managing FGTB can be simply optimized according to age category of a patient. Six-month ATT 
makes more benefits for older patients especially those with comorbidity of immune-deficient diseases. Nine-month ATT using daily strategy is a better option for younger patients at reproductive age.

\section{Abbreviations}

FGTB: Female genital tuberculosis; EPTB:Extra-pulmonary tuberculosis; MTB:Mycobacterium tuberculosis; PTB:Pulmonary tuberculosis; DS-TB:Drug-susceptible tuberculosis; DR-TB:Drug-resistant tuberculosis; ATT:Antituberculosis therapy; DOT:Directly observed therapy; TBP:Tuberculous peritonitis; TST:Tuberculin Skin Test; CT:Computerized tomography; ADA:Adenosine deaminase; AFB:Acid-fast bacilli; TRS:Tuberculin reaction size; DILI:drug-induced liver injury.

\section{Declarations}

\section{Availability of data and materials}

All the dataset generated or analyzed during the current study are available from the corresponding author upon reasonable request.

\section{Ethics approval and consent to participate}

Ethics approval and consent to participate were waived because it was a real world study.

\section{Consent for publication}

Not applicable.

\section{Competing interests}

The authors declare that they have no competing interests.

\section{Funding}

This study was supported by Health Ministry of Anhui province (grant numbers 2012zy60)

\section{Authors' contributions}

JY design of the work, JS and WW analyzed the data and wrote the paper, JY, JS, WW and JD treated and followed the patients. JS, WW and JD collected the data, YC, YZ and MW validated imaging data. All authors have seen and approved the final draft of the paper.

\section{Acknowledgements}


This work was supported by Health Ministry of Anhui province. We thank all the pilots that participated in this study for patient enrollment and follow-up.

\section{References}

1. Sharma JB, Sharma E, Sharma S, Dharmendra S. Female genital tuberculosis: Revisited. Indian J Med Res. 2018; 148 Suppl 1: 71-83. https://doi.org/10.4103/ijmr.IJMR_648_18

2. World Health Organization. Global Tuberculosis Report 2019. Geneva, Switzerland: WHO, 2019. https://www.who.int/tb/publications/global_report/en/. Accessed 17 October 2019.

3. Huang D, Carugno T, Patel D. Tuberculous peritonitis presenting as an acute abdomen: a case report. Am. J. Obstet. Gynecol. 2011; 205(1): e11-4. https://doi.org/ 10.1016/j.ajog.2011.01.039

4. Liu Q, Zhang Q, Guan Q, Xu JF, Shi QL. Abdominopelvic tuberculosis mimicking advanced ovarian cancer and pelvic inflammatory disease: a series of 28 female cases. Arch Gynecol Obstet. 2014; 289(3): 623-9. https://doi.org/10.1007/s00404-013-3034-2

5. Tal R, Lawal T, Granger E, et al. Genital TB screening at an academic fertility center in the US. Am. J. Obstet. Gynecol. 2020 (accepted). https://doi.org/10.1016/j.ajog.2020.05.045

6. World Health Organization. Guidelines for treatment of drug-susceptible tuberculosis and patient care. Geneva, Switzerland: WHO, 2017. https://www.who.int/tb/publications/2017/dstb_guidance_2017/en/. Accessed 25 May 2017.

7. Gillespie S, Crook A, McHugh T, et al. Four-month moxifloxacin-based regimens for drug-sensitive tuberculosis. N. Engl. J. Med. 2014; 371(17): 1577-87. https://doi.org/ 10.1056/NEJMoa1407426

8. Johnson J, Hadad D, Dietze R, et al. Shortening treatment in adults with noncavitary tuberculosis and 2-month culture conversion. Am. J. Respir. Crit. Care Med. 2009; 180(6): 558-63. https://doi.org/10.1164/rccm.200904-05360C

9. Swaminathan S, Narendran G, Venkatesan P, et al. Efficacy of a 6-month versus 9-month intermittent treatment regimen in HIV-infected patients with tuberculosis: a randomized clinical trial. Am. J. Respir. Crit. Care Med. 2010; 181(7): 743-51. https://doi.org/ 10.1164/rccm.200903-04390C

10. Merle $\mathrm{C}$, Fielding $\mathrm{K}$, Sow $\mathrm{O}$, et al. A four-month gatifloxacin-containing regimen for treating tuberculosis. N. Engl. J. Med. 2014; 371(17): 1588-98. https://doi.org/ 10.1056/NEJMx150015

11. Makharia G, Ghoshal U, Ramakrishna B, et al. Intermittent Directly Observed Therapy for Abdominal Tuberculosis: A Multicenter Randomized Controlled Trial Comparing 6 Months Versus 9 Months of Therapy. Clin. Infect. Dis. 2015; 61(5): 750-7. https://doi.org/ 10.1093/cid/civ376

12. Sharma JB, Singh N, Dharmendra S, et al. Six months versus nine months anti-tuberculous therapy for female genital tuberculosis: a randomized controlled trial. Eur J Obstet Gynecol Reprod Biol. 2016; 203: 264-73. https://doi.org/ 10.1016/j.ejogrb.2016.05.035

13. Lee L, Chou C, Wang J, et al. Enzyme-linked immunospot assay for interferon-gamma in the diagnosis of tuberculous pleurisy. Clin Microbiol Infect. 2009; 15(2): 173-9. https://doi.org/ 10.1111/j.1469-0691.2008.02655.x 
14. Liu XQ, Bian SN, Cheng $X \mathrm{H}$, et al. Utility of T-cell interferon- $\gamma$ release assays for the diagnosis of female genital tuberculosis in a tertiary referral hospital in Beijing, China. Medicine. 2016; 95(44): e5200. https://doi.org/10.1097/MD.0000000000005200

15. Caliskan E, Cakiroglu Y, Sofuoglu K, Doger E, Akar ME, Ozkan SO. Effects of salpingectomy and antituberculosis treatments on fertility results in patients with genital tuberculosis. J Obstet Gynaecol Res. 2014; 40(10): 2104-9. https://doi.org/ 10.1111/jog.12450

16. Sharma J. Current Diagnosis and Management of Female Genital Tuberculosis. J Obstet Gynaecol India. 2015; 65(6): 362-71. https://doi.org/ 10.1007/s13224-015-0780-z

17. Pettit AC, Cummins J, Kaltenbach LA, Sterling TR, Warkentin JV. Non-adherence and drug-related interruptions are risk factors for delays in completion of treatment for tuberculosis. Int $\mathrm{J}$ Tuberc Lung Dis. 2013; 17(4): 486-92. https://doi.org/ 10.5588/ijtld.12.0133

18. Tiberi S, du Plessis N, Walzl G, et al. Tuberculosis: progress and advances in development of new drugs, treatment regimens, and host-directed therapies. Lancet Infect Dis. 2018; 18(7): e183-e98. https://doi.org/ 10.1016/S1473-3099(18)30110-5

19. van den Boogaard J, Boeree M, Kibiki G, Aarnoutse R. The complexity of the adherence-response relationship in tuberculosis treatment: why are we still in the dark and how can we get out? Trop. Med. Int. Health. 2011; 16(6): 693-8. https://doi.org/ 10.1111/j.1365-3156.2011.02755.x

20. Bahadur A, Malhotra N, Mittal S, Singh N, Gurunath S. Second-look hysteroscopy after antitubercular treatment in infertile women with genital tuberculosis undergoing in vitro fertilization. Int $\mathrm{J}$ Gynaecol Obstet. 2010; 108(2): 128-31. https://doi.org/ 10.1016/j.ijgo.2009.08.031

\section{Tables}




\begin{tabular}{|c|c|c|c|}
\hline Characteristics & $\begin{array}{l}\text { Group A } \\
(n=41)\end{array}$ & $\begin{array}{l}\text { Group B } \\
(n=68)\end{array}$ & $\begin{array}{c}P \\
\text { Value } \\
\end{array}$ \\
\hline \multicolumn{4}{|l|}{ Epidemiological characteristics, n (\%) } \\
\hline Age, mean and range (years) & $27.7(18-34)$ & $55.9(37-81)$ & 0.000 \\
\hline Low socialeconomical status & $30(73.2)$ & $54(79.4)$ & N.S. \\
\hline Comorbidity with immuno-deficient diseases" & $1(2.4)$ & $11(16.2)$ & 0.057 \\
\hline Pregnancy/reproduction in the past 1 year & $13(31.7)$ & $2(2.9)$ & 0.000 \\
\hline Previous non-reproductive gynecologic surgeries & $2(4.9)$ & $6(8.8)$ & N.S. \\
\hline Previous infection of TB or contact with TB & $9(22.0)$ & $15(22.1)$ & N.S. \\
\hline Menorthagia or secondary amenorrhea & $11(26.8)$ & $18(26.5)$ & N.S. \\
\hline \multicolumn{4}{|l|}{ Clinical characteristics, n (\%) } \\
\hline Abdominal pain or distension & $33(80.5)$ & $59(86.8)$ & N.S. \\
\hline Abdominal mass & $12(29.3)$ & $18(26.5)$ & N.S. \\
\hline Infertility & $15(36.6)$ & $8(11.8)$ & 0.002 \\
\hline Fever and/or night sweat & $12(29.3)$ & $12(17.6)$ & N.S. \\
\hline Weight Loss & $11(26.8)$ & $15(22.1)$ & N.S. \\
\hline Cough & $5(12.2)$ & $9(13.2)$ & N.S. \\
\hline Moderate-to-massive ascites & $9(22.0)$ & $41(60.3)$ & 0.000 \\
\hline \multicolumn{4}{|l|}{ Laboratory characteristics, n (\%) } \\
\hline Anemia $(\mathrm{Hb}<11 \mathrm{~g} / \mathrm{dL})$ & $21(51.2)$ & $36(52.9)$ & N.S. \\
\hline Low albumin $(<3.5 \mathrm{~g} / \mathrm{dL})$ & $20(48.8)$ & $32(47.1)$ & N.S. \\
\hline Raised ESR & $32(78.0)$ & $47(69.1)$ & N.S. \\
\hline Raised serum CA-125 $(>35 \mathrm{v} / \mathrm{mL})$ & $36(87.8)$ & $59(86.8)$ & N.S. \\
\hline \multicolumn{4}{|l|}{ Ascitic examinations } \\
\hline Raised total protein $(>2.5 \mathrm{~g} / \mathrm{dL})^{c}$ & $15(88.2)$ & $36(85.7)$ & N.S. \\
\hline Raised white blood counts $\left(>250 / \mathrm{mm}^{3}\right)^{c}$ & $14(82.4)$ & $38(90.5)$ & N.S. \\
\hline Significant raised levels of ADA $(>35 \mathrm{w} / \mathrm{L})^{\mathrm{c}}$ & $15(88.2)$ & $20(47.6)$ & 0.010 \\
\hline Positive result of AFB smeariculture ${ }^{d}$ & $3(20.0)$ & $3(13.0)$ & N.S. \\
\hline Positive result of AFB smear/culture from sputum " & $5(13.9)$ & $3(10.7)$ & N.S. \\
\hline Moderate-to-strong positive $\operatorname{TST}^{f}$ & $8(66.7)$ & 7 (23.3) & 0.022 \\
\hline Moderate-to-strong positive T.SPOTIB ${ }^{3}$ & $27(96.4)$ & $24(64.9)$ & 0.006 \\
\hline
\end{tabular}

$\mathrm{ADA}$, adenosine deaminase; $\mathrm{AFB}$, acid-fast bacilli; $\mathrm{ESR}$, erythrocyte sedimentation rate; $\mathrm{Hb}$, hemoglobin; N.S., not statistically significant; TB, tuberculosis; TST, Tuberculin Skin Test.

"included diabetes, liver cirrhos is and autoimmme dis eases; "included cesarean delivery, natural delivery, spontaneous abortion and ampullary pregnancy; "examined in 17 of 41 and 42 of 68 in 2 groups respectively; ${ }^{\text {an }}$ 15 of 41 and 23 of 68 respectively, ${ }^{\circ}$ in 36 of 41 and 28 of 68 respectively, ${ }^{f}$ in 12 of 41 and 30 of 68 respectively, ${ }^{3}$ in 28 of 41 and 37 of 68 respectively. 
Table 2. Predominant Surgical and Histopathological Findings among 41 patients at Baseline

\begin{tabular}{|c|c|c|c|}
\hline Findings & $\begin{array}{l}\text { Group A } \\
(n=17)\end{array}$ & $\begin{array}{l}\text { Group B } \\
(\mathrm{n}=27)\end{array}$ & $\begin{array}{c}P \\
\text { Value }\end{array}$ \\
\hline \multicolumn{4}{|l|}{ Laparotomic or Laparoscopic findings, $\mathrm{n}(\%)$} \\
\hline Normal & $2(11.8)$ & $5(18.5)$ & N.S. \\
\hline Thickening-stiff tubes/tortuous tubes & $13(76.5)$ & $9(33.3)$ & 0.013 \\
\hline Hydrosalpinx or pyosalpinx & $5(29.4)$ & $1(3.7)$ & 0.049 \\
\hline Encapsulated tubes and/or ovaries & $2(11.8)$ & $2(7.4)$ & N.S. \\
\hline Cyst tubes/Granuloma tubes & $1(5.9)$ & $5(18.5)$ & N.S. \\
\hline Cyst/bulky ovaries & $2(11.8)$ & $2(7.4)$ & N.S. \\
\hline Miliary tubercles & $11(64.7)$ & $15(55.6)$ & N.S. \\
\hline Pelvic adhesions & $14(82.4)$ & $12(44.4)$ & 0.030 \\
\hline \multicolumn{4}{|l|}{ Histopathological findings, $n(\%)$} \\
\hline Granulomas of Langhans giant cells & $3(17.6)$ & $9(33.3)$ & N.S. \\
\hline $\begin{array}{l}\text { Granulomas of Langhans giant cells and caseous } \\
\text { necrosis }\end{array}$ & $14(82.4)$ & $17(63.0)$ & N.S. \\
\hline Massive lymphatic infiltration & $0(0)$ & $1(3.7)$ & N.S. \\
\hline
\end{tabular}

N.S., not statistically significant.

\section{Table 3. Predominant Site of the Disease and Comorbidity of other types of TB at Baseline}

\begin{tabular}{|c|c|c|c|}
\hline Composition & $\begin{array}{c}\text { Group A } \\
(\mathrm{n}=41)\end{array}$ & $\begin{array}{c}\text { Group B } \\
(n=68)\end{array}$ & $\begin{array}{c}P \\
\text { Value } \\
\end{array}$ \\
\hline \multicolumn{4}{|c|}{ Predominant site of the Disease, $\mathrm{n}(\%)$} \\
\hline Female gential organs & $6(14.6)$ & $7(10.3)$ & N.S. \\
\hline Peritoneum & $5(12.2)$ & $29(42.6)$ & 0.004 \\
\hline Mixed & $30(73.2)$ & $32(47.1)$ & N.S. \\
\hline \multicolumn{4}{|c|}{ Comorbidity of PTB, n (\%) } \\
\hline Non-active PTB & $7(17.1)$ & $22(32.4)$ & N.S. \\
\hline Active PTB/mixed & $22(53.7)$ & $17(25.0)$ & 0.009 \\
\hline Overall & $29(70.7)$ & $39(57.4)$ & N.S. \\
\hline \multicolumn{4}{|c|}{ Comorbidity of other EPTB, $n(\%)$} \\
\hline Intestinal TB & $5(12.2)$ & $6(8.8)$ & N.S. \\
\hline Liver TB & $3(7.3)$ & $3(4.4)$ & N.S. \\
\hline Splenic TB & $0(0)$ & $5(7.4)$ & N.S. \\
\hline Renal TB & $0(0)$ & $3(4.4)$ & N.S. \\
\hline Overal & $8(19.5)$ & $17(25.0)$ & N.S. \\
\hline
\end{tabular}

EPTB, extrapulmonary tuberculosis; N.S., not statistically significant; PTB, pulmonary tuberculosis; TB, tuberculosis. 
Table 4. Results of Compliance and Side effects during 9-month period since the Initiation of ATT : Intent-to-Treat Analysis

\begin{tabular}{|l|c|c|c|}
\hline Outcome & $\begin{array}{c}\text { Group A } \\
(\mathrm{n}=41)\end{array}$ & $\begin{array}{c}\text { Group B } \\
(\mathrm{n}=68)\end{array}$ & $\begin{array}{c}P \\
\text { vaule }\end{array}$ \\
\hline Poor compliance & & & \\
\hline$\quad$ Irregular protocol & $1(2.4)$ & $11(16.2)$ & 0.056 \\
\hline $\begin{array}{l}\text { Lost to follow up } \\
\quad \text { Overall }\end{array}$ & $2(4.9)$ & $7(10.3)$ & N.S. \\
\hline Side effects & $3(7.3)$ & $18(26.5)$ & 0.027 \\
\hline$\quad$ Anorexia, $\mathrm{n}(\%)$ & $5(12.2)$ & $18(26.5)$ & 0.076 \\
\hline Vomiting, $\mathrm{n}(\%)$ & $3(7.3)$ & $10(14.7)$ & N.S. \\
\hline Drug-induced liver injury, $\mathrm{n}(\%)$ & $2(4.9)$ & $14(20.6)$ & 0.049 \\
\hline Leucopenia/thrombocytopenia, $\mathrm{n}(\%)$ & $1(2.4)$ & $5(7.4)$ & N.S. \\
\hline$\quad$ Overall single side effects, $\mathrm{n}(\%)$ & $7(17.1)$ & $29(42.6)$ & 0.006 \\
\hline
\end{tabular}

N.S., not statistically significant.

Table 5. Results of Efficacay and Side Effects at two Observation Time-Points: Per-Protocol Analysis

\begin{tabular}{|c|c|c|c|c|c|c|}
\hline \multirow[t]{2}{*}{ Efficacy and side effects } & \multicolumn{2}{|c|}{ Group A } & \multirow{2}{*}{$\begin{array}{c}P \\
\text { vaule }\end{array}$} & \multicolumn{2}{|c|}{ Group B } & \multirow{2}{*}{$\begin{array}{c}P \\
\text { vaule }\end{array}$} \\
\hline & $\begin{array}{c}6 \text { months } \\
(\mathrm{n}=38)\end{array}$ & $\begin{array}{c}9 \text { months } \\
(\mathrm{n}=38)\end{array}$ & & $\begin{array}{c}6 \text { months } \\
(\mathrm{n}=57)\end{array}$ & $\begin{array}{c}9 \text { months } \\
(\mathrm{n}=50)\end{array}$ & \\
\hline \multicolumn{7}{|l|}{ Clinical response, $\mathrm{n}(\%)$} \\
\hline Complete response & $24(63.2)$ & $35(92.1)$ & 0.006 & $50(87.7)$ & $44(88.0)$ & N.S. \\
\hline Partial response & $12(31.6)$ & $2(5.3)$ & 0.008 & $5(8.8)$ & $4(8.0)$ & N.S. \\
\hline No response & $2(5.3)$ & $1(2.6)$ & N.S. & $2(3.5)$ & $2(4.0)$ & N.S. \\
\hline \multicolumn{7}{|l|}{ Side effects, $\mathrm{n}(\%)$} \\
\hline Anorexia & $2(5.3)$ & $4(10.5)$ & N.S. & $5(8.8)$ & $13(26.0)$ & 0.016 \\
\hline Vomiting & $1(2.6)$ & $2(5.3)$ & N.S. & $3(5.3)$ & $7(14.0)$ & N.S. \\
\hline Drug-induced liver injury & $2(5.3)$ & $3(7.9)$ & N.S. & $4(7.0)$ & $11(22.0)$ & 0.033 \\
\hline Drug rash & $1(2.6)$ & $0(0)$ & N.S. & $2(3.5)$ & $1(2.0)$ & N.S. \\
\hline Leucopenia/thrombocytopenia & $0(0)$ & $1(2.6)$ & N.S. & $2(3.5)$ & $3(6.0)$ & N.S. \\
\hline Overall single side effects & $4(10.5)$ & $6(15.8)$ & N.S. & $13(22.8)$ & $21(42.0)$ & 0.033 \\
\hline
\end{tabular}

N.S., not statistically significant.

\section{Figures}




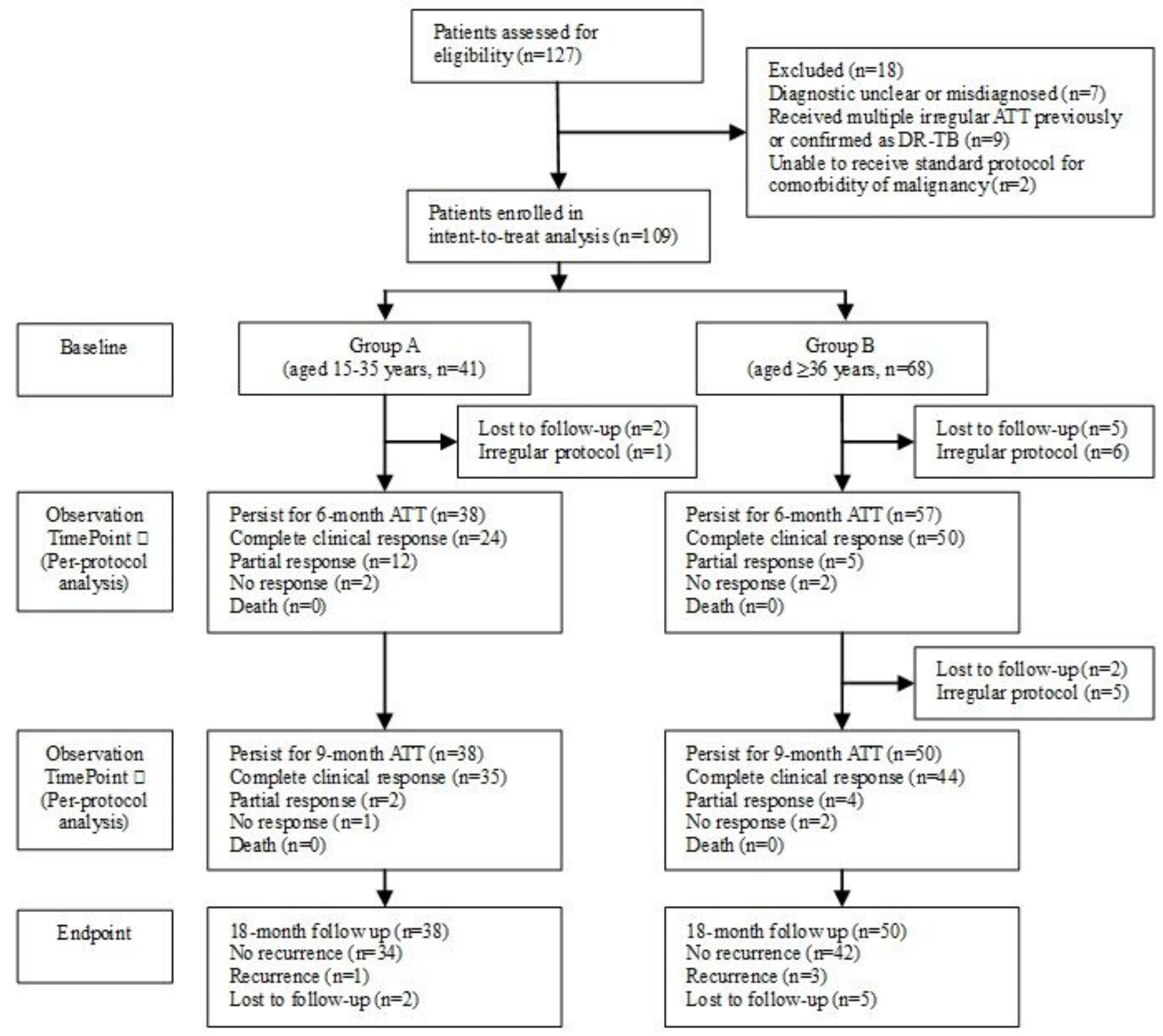

\section{Figure 1}

Flow Chart of Patients with female genital tuberculosis or tuberculous peritonitis being followed at the Department of Infectious Diseases, the First Affiliated Hospital of Wanan Medical College, Wuhu, P.R. China Abbreviations: ATT, anti-tuberculosis therapy; DR-TB, drug-resistant tuberculosis 